\title{
BMJ Open Serogroup-specific meningococcal carriage by age group: a systematic review and meta-analysis
}

\author{
Meagan E Peterson, ${ }^{\oplus 1}$ You Li, ${ }^{\oplus 1}$ Heather Shanks, ${ }^{1}$ Rebecca Mile, ${ }^{1}$ Harish Nair, ${ }^{2}$ \\ Moe H Kyaw, ${ }^{3}$ on behalf of Meningococcal Carriage Group
}

To cite: Peterson ME, Li Y, Shanks H, et al. Serogroupspecific meningococcal carriage by age group: a systematic review and meta-analysis. BMJ Open 2019;9:e024343. doi:10.1136/ bmjopen-2018-024343

- Prepublication history and additional material for this paper are available online. To view these files, please visit the journal online (http://dx.doi. org/10.1136/bmjopen-2018024343).

Received 23 May 2018 Revised 3 December 2018 Accepted 26 February 2019

D Check for updates

C Author(s) (or their employer(s)) 2019. Re-use permitted under CC BY-NC. No commercial re-use. See rights and permissions. Published by BMJ.

${ }^{1}$ Centre for Global Health Research, University of Edinburgh School of Molecular Genetic and Population Health Sciences, Edinburgh, UK ${ }^{2}$ University of Edinburgh School of Molecular Genetic and Population Health Sciences, Edinburgh, UK

${ }^{3}$ Sanofi Pasteur, Inc., Swiftwater, Pennsylvania, USA

Correspondence to

Dr Harish Nair;

harish.nair@ed.ac.uk

\section{ABSTRACT}

Objective Neisseria meningitidis carriage prevalence has known variation across the lifespan, but it is unclear whether carriage varies among meningococcal capsular groups. Therefore, we aimed to characterise group-specific meningococcal carriage by age group and world region from 2007 to 2016.

Design Systematic review and meta-analysis.

Data sources MEDLINE, Embase, Global Health

Database, WHO Global Health Library, Web of Science, Current Contents Connects, China National Knowledge Infrastructure and Wanfang were systematically searched. Database searches were conducted through July 2018 and Google Scholar forward searches of included studies were conducted through August 2018. References of included studies and relevant conference abstracts were also searched to identify additional articles for inclusion. Eligibility criteria Studies were eligible for inclusion if they reported capsular group-specific meningococcal carriage in a healthy population of a specified age group and geographical region. For this review, only studies conducted between 2007 and 2016 were included.

Data extraction and synthesis Data were independently extracted by two authors into Microsoft Access. Studies were assessed for risk of bias using the Joanna Briggs Institute Critical Appraisal Checklist for Studies Reporting Prevalence Data. Studies eligible for inclusion in quantitative analyses by pre-specified age groups were pooled using random effects meta-analyses. Results are reported by capsular group, age group and WHO region. Where meta-analyses were not appropriate, study results were discussed narratively.

Results 7511 articles were identified and 65 were eligible for inclusion. Adolescents and young adults were the focus of many studies ( $n=24)$, especially in the Americas and Europe. Studies from China and Africa, typically, included data from a wider age range. The overall carriage prevalence varied markedly by age group and region. Based on the available data, 21 studies were included in meta-analyses reporting serogroup carriage for: all ages in Africa, 18-24-year olds in the Americas, and 11-17 and 18-24-year olds in Europe. Capsular groups W, X, Y and 'other' (non-ABCWXY, including non-groupable) were the most prevalent in Africa, and 5-17-year olds had higher carriage prevalence than other age groups. 'Other' serogroups $(11.5 \%, 95 \% \mathrm{Cl} 1.6 \%$ to $16.1 \%)$ were the most common among 18-24-year olds from the Americas. In Europe, 18-24-year old were carriers more frequently than

\section{Strengths and limitations of this study}

This is the first review investigating serogroup-specific meningococcal carriage by age group.

- A comprehensive literature review was performed with additional granular data requested from authors for meta-analyses.

- Meta-analyses were conducted for all ages in Africa, 18-24-year olds in the Americas and 11-17year olds and 18-24-year-old in Europe.

- Other regions and age groups were synthesised narratively due to differences in reported age groups or paucity of data.

11-17-year olds, and groups $\mathrm{B}(5.0 \%, 95 \% \mathrm{Cl} 3.0 \%$ to $7.5 \%), \mathrm{Y}(3.9 \%, 95 \% \mathrm{Cl} 1.3 \%$ to $7.8 \%)$ and 'other' $(6.4 \%$, $95 \% \mathrm{Cl} 3.1 \%$ to $10.8 \%$ ) were the most commonly carried in the older age group.

Conclusions of the age groups included in the analysis, carriage patterns by age were similar across capsular groups within a region but differed between regions. Data gaps remain for age- and capsular group-specific carriage in many regions, especially in the Eastern Mediterranean and South-East Asia. As such, clear and robust conclusions about the variation of capsular group-specific carriage by age group and WHO region were unable to be determined. PROSPERO registration number CRD42017074671.

\section{BACKGROUND}

Invasive meningococcal disease (IMD) is a severe bacterial infection caused by Neisseria meningitidis. IMD most commonly presents as meningitis and septicemia, and despite advances in medical care, continues to have a case fatality rate of approximately $10 \%-15 \%$ and debilitating sequelae in $10 \%-15 \%$ of survivors. ${ }^{12}$ The incidence of IMD varies globally, with the highest burden in the African meningitis belt and in young children and adolescents. ${ }^{3}$ Historically, pharyngeal carriage of the bacterium was estimated to occur in approximately $10 \%$ of the general population at any one point in time, with most people becoming carriers at multiple points in their 
lifetime. ${ }^{4}$ Recent studies estimate that carriage prevalence peaks during late adolescence in Europe and early adolescence in Africa. ${ }^{56}$ As such, persons in these age groups are likely to be important transmitters of the disease in these regions.

There are 12 capsular groups of N. meningitidis, six of which cause the majority of disease globally-A, B, C, W, $\mathrm{X}$ and $\mathrm{Y}$. Currently, vaccines are available targeting five of the 12 serogroups: polysaccharide and conjugate vaccines for serogroups A, C, W and Y, and broad protection recombinant protein-based vaccines targeting serogroup B. ${ }^{7}$ Since IMD is primarily transmitted through carriage, to inform appropriate vaccine policies, it is important to understand the serogroups being carried and if these serogroups differ by age group. Conjugate vaccines can effectively induce indirect herd immunity through clearing carriage, at least for serogroup $\mathrm{C}$, as seen in the United Kingdom post-MenC vaccine introduction. ${ }^{78}$

Although reviews and analyses have been conducted to determine the overall carriage prevalence by age, no review has attempted to quantify serogroup-specific carriage in different age groups. ${ }^{56}$ Understanding the age group with the highest level of carriage, and which serogroups they carry, is important to better understand disease transmission and determine optimal target ages for vaccination. ${ }^{79}$ As countries introduce vaccination policies targeting age groups with high overall carriage levels, it is important to understand if other age groups could have a low overall carriage prevalence yet be important carriers of certain capsular groups, and, thus, disease transmitters and source of potential outbreaks. Therefore, we aimed to analyse carriage studies conducted in persons of defined ages from 2007 to 2016 in order to elucidate any differences in the carriage of meningococcal serogroups by age group.

\section{METHODS}

\section{Search strategy and data sources}

We conducted a systematic review of the literature to identify meningococcal carriage studies in specific age groups conducted between 1st January 2007 and 31st December 2016 (PROSPERO number CRD42017074671). Two authors (MP and HS) searched six databases (MEDLINE, Embase, Global Health Database, WHO Global Health Library, Web of Science and Current Contents Connects). Additionally, a native speaker (YL) searched two Chinese literature databases (China National Knowledge Infrastructure and Wanfang). Search terms were developed for MEDLINE (online supplementary table 1) and adapted for the remaining databases. Google Scholar forward citations, relevant conference abstract lists, and references of identified studies and reviews were also searched. Database searches were last conducted in July 2018 and Google Scholar searches in August 2018. We did not exclude any article based on language, and Google Translate was used to assist with screening. If questions remained, we sought translation assistance.

\section{Inclusion and exclusion criteria}

Studies were considered for inclusion if they reported capsular group-specific pharyngeal carriage in a healthy population of a defined age group, provided clear geographical location of participants and were reasonably representative of the general population. If the age of participants was not reported but referred to as a generic age group (eg, adolescents, university students, elementary students, etc), the exact ages were requested from the authors.

Studies were ineligible for inclusion if they only provided disease data or were carriage studies among cases or close-contacts of cases. Studies that had unclear reporting of serogroups or participants sampled, unclear geographical location or year, only tested for one serogroup or serogrouped/reported $<75 \%$ of positive carriage specimens were also excluded. Studies reporting secondary data, case studies, commentaries or reviews were also ineligible for inclusion.

\section{Data collection and management}

Two authors (MP and HS) independently screened the titles and abstracts of all studies and the relevant full-text articles. Studies selected for inclusion were independently extracted into Microsoft Access by two authors (MP and YL) using a piloted extraction template. One author screened and extracted the Chinese articles (YL) with input from another (MP). Any disagreement in eligibility or extractions was discussed and a consensus reached before proceeding.

\section{Quality assessment}

The included studies were assessed for quality using a modified Joanna Briggs Institute Checklist for Prevalence Studies and given a low, medium, or high risk-of-bias score. ${ }^{10}$ Factors assessed included the sampling framework and recruitment methods, calculation of adequate sample size, and detailed description of study methodologies and population. Since no methodological 'gold standard' exists, the validity of the methodologies used was not assessed.

\section{Data analysis}

In order to have standardised age groups for meta-analyses, we requested detailed data from the authors of eligible studies. The age groups were pre-specified based on life stage as follows (in years): $0-4,5-10,11-17$, 18-25, 26-29, 30-49, 50-64 and $\geq 65$. Serogroup-specific meta-analyses for serogroups A, B, C, W, X, Y and 'other' (non-ABCWXY, including non-groupable) were conducted stratified by age group and WHO region if data from at least three studies were available for all serogroups in a given age group and WHO region. We conducted all analyses in StataIC 13 (64-bit) using the metaprop command and reported the results using a random-effects model with the Freeman-Tukey transformation and exact binomial CIs (StataCorp, 2013) ${ }^{11}$ Since sample sizes for multiple covariates were expected to be 


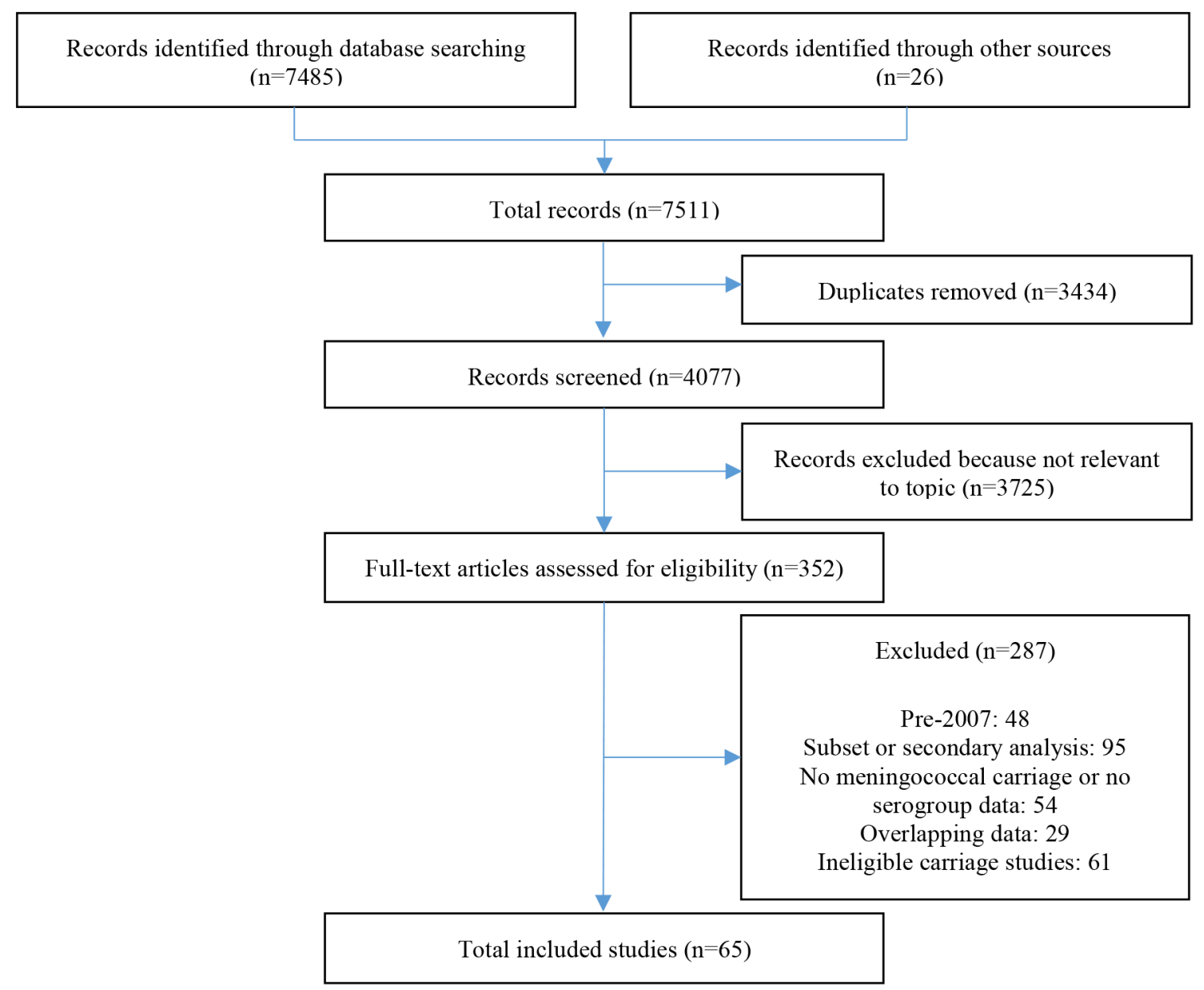

Figure 1 Preferred Reporting Items for Systematic Reviews and Meta-Analyses flowchart of included studies.

small and there were insufficient studies, meta-regression was not considered feasible. Heterogeneity was measured using $\mathrm{I}^{2}$ values, and classified as low $\left(\mathrm{I}^{2}<50 \%\right)$, moderately high $\left(50 \% \leq \mathrm{I}^{2}<75 \%\right)$ or high $\left(\mathrm{I}^{2} \geq 75 \%\right)$.

Multi-site studies performed at the same time point were combined so that each data point represented one country and one time point. Only baseline data were included from longitudinal cohort studies or repeat cross-sectional studies with a nested cohort. Studies with a swabbed sample size $<10$ in a particular age group were not included in the analyses $(n=2)$ due to the use of the Freeman-Tukey transformation and its degradation with extremely sparse data. ${ }^{11}$ If a study included multiple methodology comparisons (eg, nasopharyngeal vs oropharyngeal swab, slide agglutination vs PCR), the results from one method were included in the review. The results from the methodology comparison studies and the notation of which methodology was included in the review are detailed in online supplementary table 2 .

\section{Patient involvement}

No patients were involved in setting the research agenda for this review.

\section{RESULTS}

\section{Study characteristics}

Sixty-five studies reporting serogroup-specific carriage among a defined age group met our inclusion criteria (figure 1). Forty-three were one-time cross-sectional studies, 12 were repeat cross-sectional studies, five were repeat cross-sectional studies with a nested cohort (either intentional or unintentional), four were cohort studies and one was a randomised controlled trial. Almost half of the studies were from China, and the rest were from Americas or Europe. However, studies with the largest sample sizes were from the African region with an average of 3282 participants sampled per country per year. Study populations varied in their coverage of MenB, MenA, MenACWY and MenC vaccinations (online supplementary table 3).

Swabbing and laboratory methods varied between studies. Fifty-five studies reported swabbing the "posterior pharynx', 'oropharynx' or 'pharynx,' while nine swabbed the 'nasopharynx' and one swabbed both the sites in order to compare the methodology. To characterise the capsular group, 20 studies used agglutination methods alone, 34 used genetic methods (eg, PCR, whole genome sequencing) alone or to supplement agglutination and 11 did not report the method used. 
Thirteen studies reported the results of multiple methodologies for detecting the meningococcus or grouping the carriage samples (online supplementary table 2). Pharyngeal swabbing was more sensitive than nasopharyngeal swabbing. Additionally, genotypic methods of capsular group characterisation were more sensitive and specific than phenotypic methods. However, results from direct swab PCR were less conclusive, and due to varying methodologies in studies, assessment of its sensitivity and specificity cannot be definitively assessed in this review. In most cases, it was more sensitive than isolate PCR but potentially less specific. However, it should be noted that a variety of PCR primers for identifying $N$. meningitidis were used for cultured isolates across studies.

Most included studies received a medium risk-of-bias score, mostly because study population characteristics were not reported (online supplementary table 4). No study was excluded from the review based on the quality score, and no study received a high risk-of-bias score to necessitate the pre-specified sensitivity analysis. However, the results further indicated a need for clear reporting of methodology and standardisation.

\section{Carriage by WHO region}

In the African region, all studies were from countries located in the meningitis belt. Most studies included both children and adults (at least to age 29). The overall carriage prevalence was, typically, lower than other WHO regions and ranged from $1.7 \%$ among 1-29-year olds in Burkina Faso to $13.2 \%$ among 10-18-year olds in the Gambia. 'Other' serogroups were less frequently identified compared with other regions. Depending on the year, serogroup $\mathrm{W}, \mathrm{X}$ or $\mathrm{Y}$ was the most commonly carried disease-causing serogroup in Burkina Faso, and serogroup $\mathrm{W}$ was the most common in countries in the rest of the region (online supplementary table 5).

In the region of the Americas, studies were almost exclusively conducted among adolescents and university students. The overall carriage prevalence ranged from $4.0 \%$ in Chilean university students to $71.1 \%$ in Brazilian university students. 'Other' serogroups were the most commonly carried in all studies, except one. Among serogroups A, B, C, W, X and Y, B was the most prevalent across the region. Serogroups $\mathrm{C}$ and $\mathrm{Y}$ were identified as the most common for select years in Latin America.

Only one study was identified from the Eastern Mediterranean region. Iranian students, 7-19 years old, had an overall carriage prevalence of $8.9 \%$, with the highest carriage among 15-19-year olds. The vast majority of carriage was of serogroup $\mathrm{C}$, with lower prevalence of serogroup B found.

As in the Americas, most studies from the European region focused on adolescents and university students. The overall carriage prevalence ranged from $5.3 \%$ in Italian 14-21-year olds to $61.9 \%$ in English university students. Serogroups B, Y and 'other' were the most prominent across the majority of the region. However, serogroup $\mathrm{W}$ was the most commonly carried in Turkey and was a prominent serogroup identified in a 2015-2016 carriage study in England.

In the South-East Asia region, only one study among Indian university students was eligible for inclusion, and only low levels of serogroup B carriage were identified.

The vast majority of studies from the Western Pacific region were from China, and, typically, included both children and adults. The overall carriage prevalence ranged from 0\% in Guangzhou in 2009 to 33.7\% in Laibin in 2011, cities in neighbouring provinces. Carriage results from China were more variable than other regions with no apparent pattern by age, year or province. The most commonly carried serogroups were A, B and C, but serogroups A, B, C, W, X, Y and 'other' were each identified as the most prominent in at least one study. Elsewhere in the region, adolescents and university students were sampled and serogroups B and 'other' were the most common.

\section{Age group meta-analysis}

In total, six studies from the African region, seven from the region of the Americas and eight from the European region were included in the meta-analyses. Only for the African region were there sufficient data for analysis for all age groups. For the regions of the Americas and Europe, analyses were restricted to the age groups for which data from at least three studies were available. There were insufficient studies or responses received from the Eastern Mediterranean, South-East Asia and Western Pacific regions for meta-analysis.

\section{Carriage across the lifespan in the African region}

There was a similar pattern of serogroup carriage across almost all age groups within Africa, and carriage estimates were the highest during childhood and adolescence (5-17year olds) (figure 2). Within the majority of age groups, the carriage of 'other' was higher than the six disease-associated serogroups. The second most commonly carried group was serogroup W, rates of which were comparable to 'other' for many age groups. Heterogeneity was high $\left(\mathrm{I}^{2} \geq 75 \%\right)$ for serogroups $\mathrm{W}, \mathrm{X}, \mathrm{Y}$ and 'other' in many age groups (online supplementary table 6 ).

\section{Carriage in 18-24-Year Olds in the region of the Americas}

Carriage estimates were extremely low $(<0.1 \%)$ for serogroups $\mathrm{A}, \mathrm{W}, \mathrm{X}$ and $\mathrm{Y}$, while 'other' had the highest carriage estimate of $11.5 \%$ (95\% CI $7.6 \%$ to $16.1 \%$ ) (figure 3). B and C represented the most prevalent of the disease-associated serogroups, with a prevalence of $1.6 \%(95 \%$ CI $0.8 \%$ to $2.6 \%)$ and $2.5 \%(<0.1 \%-7.8 \%)$, respectively. Heterogeneity was high $\left(\mathrm{I}^{2} \geq 75 \%\right)$ for serogroups B, C and 'other,' and low $\left(\mathrm{I}^{2}<50 \%\right)$ for serogroups A, W, X and Y (online supplementary table 6).

Carriage in 11-17-year olds and 18-24-year Olds in the European region

In the 11-17-year olds, serogroups A, C, W, X and Y each had $<1 \%$ prevalence (figure 4 ). Serogroup B and 'other' had a prevalence of $1.9 \%$ (95\% CI $1.3 \%$ to $2.7 \%$ ) and $3.2 \%(1.0 \%-6.6 \%)$, respectively. Heterogeneity was 


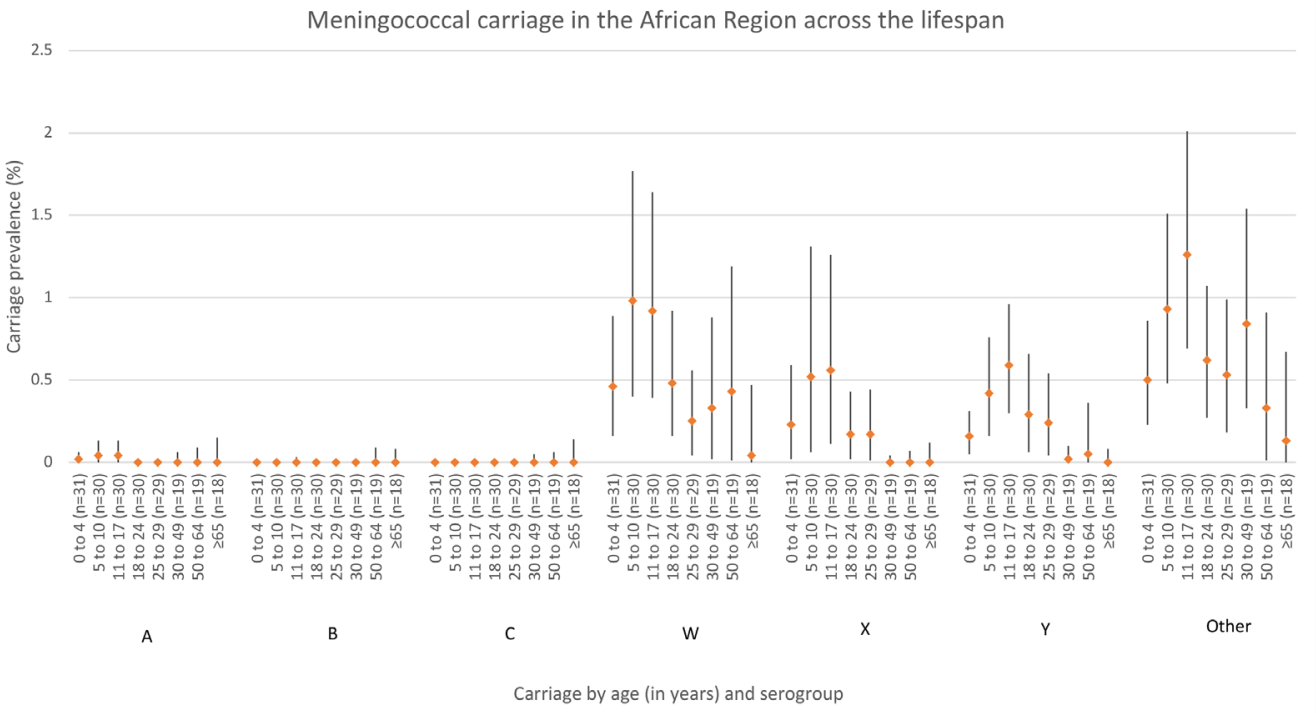

Figure 2 Meningococcal carriage in the African region across the lifespan (in years) stratified by serogroup. The number of data points included for each age meta-estimate are reported after the age group label. 'Other' includes both non-encapsulated and other capsulated (eg, E, Z).

moderately high $\left(50 \% \leq \mathrm{I}^{2}<75 \%\right)$ for serogroups $\mathrm{C}$ and $\mathrm{W}$, and high ( $\left.\mathrm{I}^{2} \geq 75 \%\right)$ for 'other'. In the 18-24-year olds, serogroups B $(5.0 \%, 95 \%$ CI $3.0 \%$ to $7.5 \%)$, Y $(3.9 \%$, 95\% CI $1.3 \%$ to $7.8 \%)$ and 'other' $(6.4 \%, 95 \%$ CI $3.1 \%$ to $10.8 \%$ ) were the most prevalent. As in the 11-17year olds, carriage of serogroups $\mathrm{A}, \mathrm{C}, \mathrm{W}$ and $\mathrm{X}$ were less common. Heterogeneity was moderately high $(50 \%$ $\left.\leq \mathrm{I}^{2}<75 \%\right)$ for serogroup $\mathrm{C}$ and high $\left(\mathrm{I}^{2} \geq 75 \%\right)$ for serogroups B, W, Y and 'other'.

\section{DISCUSSION}

To our knowledge, this is the first systematic review to explore serogroup-specific meningococcal carriage by age group globally. Additionally, our study highlights the lack of standard methodology for assessing meningococcal carriage. Since, outside of China and Africa, the majority of studies were conducted in adolescents and young adults, our understanding of serogroup-specific carriage and potential transmission in other age groups is limited. In Africa, the only region with the requisite data to investigate carriage patterns of different serogroups across age ranges, the patterns appeared similar for all serogroups where a peak was seen from childhood to adolescence (5-17-year olds). Additional multi-site studies across lifespan would be required to evaluate the lifespan dynamics in other regions.

Although the burden of IMD is high in Africa, carriage is low, with no serogroup-specific meta-estimate greater

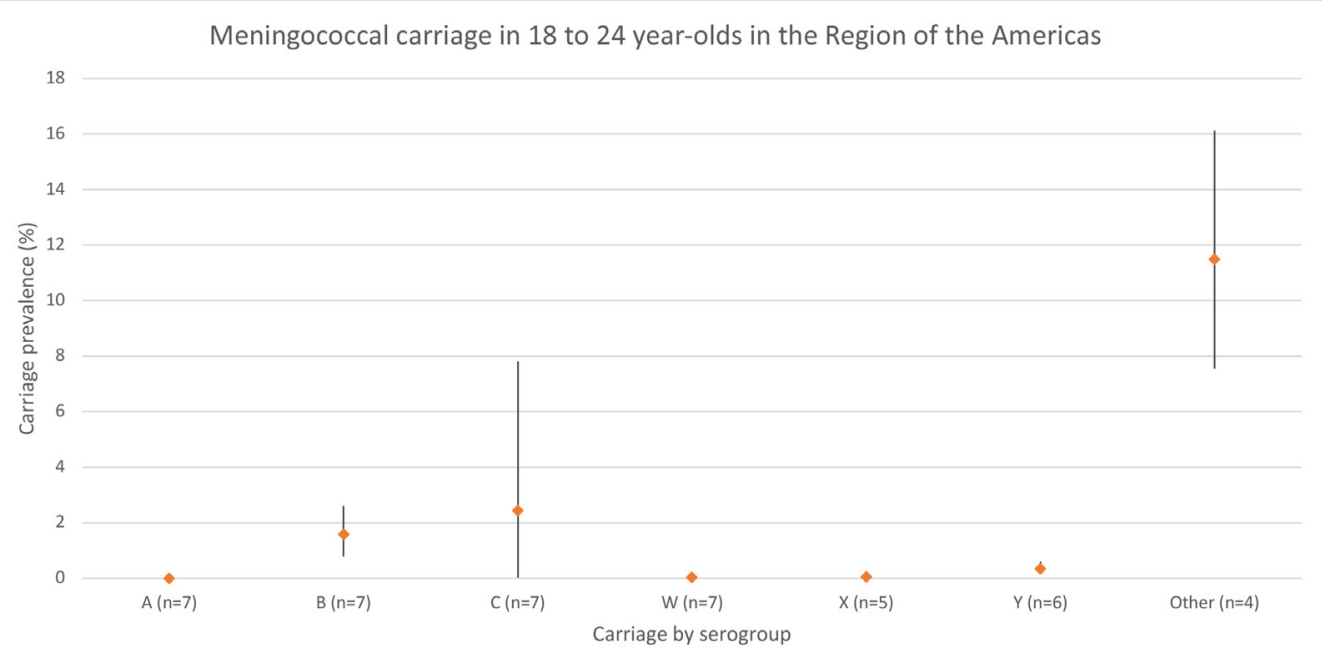

Figure 3 Meningococcal carriage in the region of the Americas in 18-24-year olds stratified by serogroup. The number of data points included for each age meta-estimate are reported after the age group label. 'Other' includes both non-encapsulated and other capsulated (eg, E, Z). 


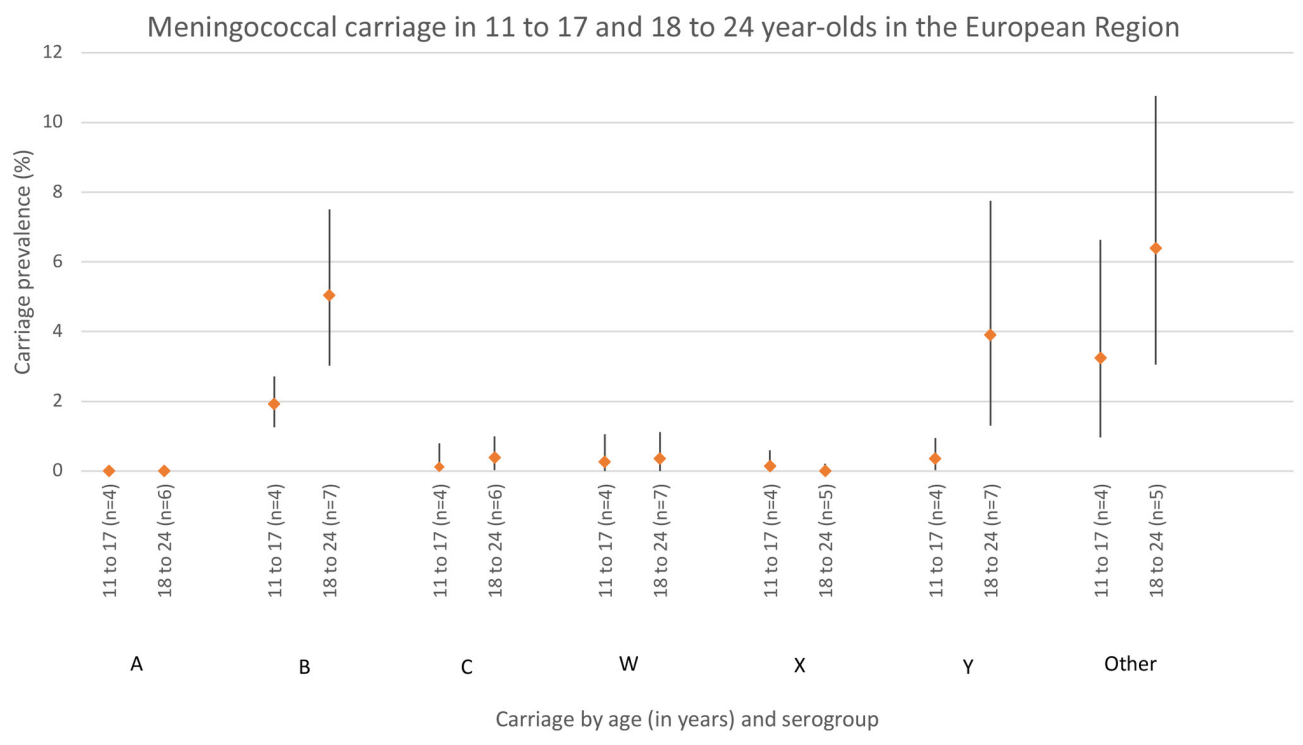

Figure 4 Meningococcal carriage in the European region in 11-17-year olds and 18-24-year olds stratified by serogroup. The number of data points included for each age meta-estimate are reported after the age group label. 'Other' includes both nonencapsulated and capsulated (eg, E, Z).

than $1.3 \%$ and the majority below $0.5 \%$. Along with the peak in carriage in 5-17-year olds, estimates for serogroups W and 'other' increased slightly among adults aged $30-64$ and $30-49$, respectively. This is, perhaps, a point for future investigation. Although serogroup Y carriage was identified, it causes few cases of IMD in the region. ${ }^{12}$ It should be noted that carriage distribution within the meningitis belt is variable and likely influenced by circulating pathogenic strains, even if the overall carriage remains low. ${ }^{13}$ The MenA campaign has dramatically decreased the disease due to serogroup $\mathrm{A}$ in the region and induced herd immunity by decreasing group A carriage, supporting introduction of MenA into national childhood immunisation schedules to provide prolonged protection against this serogroup. ${ }^{14} 15$ Consideration should also be given to expanding conjugate vaccination to other serogroups causing outbreaks in the region, such as $\mathrm{C}$ and $\mathrm{W}^{1617}$

In the Americas, the primary disease-associated serogroups carried were $\mathrm{B}$ and $\mathrm{C}$, similar to IMD serogroups in the region. ${ }^{12}$ Carriage of 'other' groups was highest in the Americas where there is a higher non-groupable carriage prevalence in North America compared with other regions. Group C-containing vaccines have been introduced in several countries in the region, which can decrease carriage of this group. While group $\mathrm{C}$ was prevalent in the regional meta-analysis, low levels of $\mathrm{C}$ carriage were identified in all studies except one, which reports a prevalence of $46 \%$. This outlier raises an important point about the sensitivity of the methods used to detect carriage, which is discussed in more detail below.

Within the European region, a high carriage prevalence of serogroups B and Y was found, which in this case is similar to the serogroups most responsible for IMD cases in the region. 'Other' groups, as expected, are proportionally more prevalent among carriers than in IMD cases. $^{18}$ A significant decrease in serogroup $\mathrm{C}$ carriage is seen after national MenC vaccination, thereby inducing herd immunity. ${ }^{8}$ All studies from the European region included in this review except one were conducted after national MenC introduction, which likely contributed to the low levels of serogroup $\mathrm{C}$ carriage identified in this review. The carriage of serogroups B, C, Y and 'other' was substantially higher in the 18-24-year olds compared with the 11-17-year olds. Acquisition of these serogroups is likely driving the increase in overall carriage, which is estimated to peak at age 19 in the region. ${ }^{6}$

The vast majority of studies among all ages from the Western Pacific region were from China, where serogroups $\mathrm{A}, \mathrm{B}$ and $\mathrm{C}$ were common. This is in line with recent increases in IMD caused by serogroup $\mathrm{B}$ and the known circulation of serogroups A and C..$^{19}$ Although polysaccharide vaccines against serogroups $\mathrm{A}$ and $\mathrm{C}$ are included in the national immunisation schedule, they do not disrupt carriage and induce herd immunity, explaining the frequent carriage of these serogroups. ${ }^{19}$ Future research is needed to elucidate when the overall carriage peaks in the region, because the determination of the primary disease transmitters would impact the targeted age group for national vaccination policies.

Because only a few studies were conducted in the Eastern Mediterranean and South-East Asia regions, or in much of the Western Pacific region, uncertainty about the prevalent serogroups being carried and how they compare to disease in these regions remains. Although current IMD serogroup data from Iran are few, serogroups B and C were identified as leading causes of meningitis cases in Tehran. ${ }^{20}$ Conversely, the one study from South-East Asia 
found university students in India to only carry serogroup $\mathrm{B}$, which rarely causes disease within the country. ${ }^{21}$ Additional studies are needed to confirm if these results are more widely generalisable to other countries within the regions.

There are several carriage studies that are either being conducted or were recently completed. These include the 'B Part of It' study among Australian adolescents and young adults (trial registries: NCT03089086, NCT03419533 and ACTRN12617000233325), the 'UKMenCar4' study among adolescents in the UK (ISRCTN72009783 and ISRCTN98439755) and a study among young adults in Japan (trial registry: JPRN-UMIN000026546). While these studies will provide valuable information, especially concerning herd immunity and invasiveness, additional studies should be considered as data gaps remain in many countries and regions.

\section{Laboratory methods and their effect on carriage detection}

How samples of $N$. meningitidis are handled and the laboratory methods used can impact carriage detection. Two literature reviews of methodology suggest that swabs taken from the posterior pharyngeal wall, and maybe the tonsils, are more sensitive than nasopharyngeal swabs or oral washes. ${ }^{22}{ }^{23}$ This is in agreement with the results from Esposito $e t$ als study included in this review that compared these techniques. ${ }^{24}$ Other factors in study design and implementation that can affect detection include the type of swab used, immediate plating versus using a transport medium and time to arrival at the laboratory for processing. ${ }^{22} 23$

Results from studies included in this review suggest that conventional microbiological methods, such as immediate plating for culture, might be less sensitive than PCR analysis of DNA taken directly from the swab or growth in Todd-Hewitt broth in some, but not all, cases. ${ }^{25-29}$ The difference in sensitivity was most noticeable for age groups with the highest carriage and 'other' groups but was sometimes important for disease-associated groups as well. This is an important consideration since carriage density is variable, the importance of which and implications for disease transmission are still being studied. ${ }^{30}$ The matter is further complicated if carriage density varies across lifespan.

How the pathogen and serogroups are determined is also important for sensitivity. Several studies from this review evaluated the use of agglutination and PCR tests to determine the serogroup or genogroup, respectively. PCR was universally more specific compared with agglutination, and sensitivity varied for isolate PCR culture versus direct swab PCR. ${ }^{27-29}$ 31-37 When using PCR for detection and genogrouping, it is important to carefully select the primers to avoid inadvertently underestimating carriage. This is especially true for non-groupable specimens as they may lack the $c t r A$ capsular transport gene, which is often used for N. meningitidis detection. As such, the addition of primers for another target, such as $\operatorname{sod} C$ and/or porA, might be appropriate. ${ }^{38} 39$
These concerns will continue to abound and make future analyses difficult. This is especially true as new techniques, such as direct PCR analysis of pharyngeal swabs as employed in studies by Gilca et al, Rizek et al, Jeppessen $e t a l$, and van Ravenhorst $e t a l$, increase in popularity. ${ }^{27-29}$ Additional research is needed to determine the most sensitive and effective methods for carriage detection and characterisation. Standardised methodologies for carriage studies would be welcomed.

However, even if a 'gold standard' methodology is agreed on, trends by capsular group and age group might not be found due to myriad other influencing factors, such as strain variability, unpredictable outbreaks, and variable risk factors for carriage. This is highlighted in the MenAfriCar study included in this review, which utilised standardised methodology in seven countries within the African Meningitis Belt. ${ }^{40}$ Even still, considerable differences are noted in carriage prevalences, at times within the same country as seen in Senegal. Reasons for this are complex and, as such, elucidation of trends by age and capsular group, if they exist, could continue to be difficult.

\section{Strengths and limitations}

Strengths of this study include the comprehensive literature review, including two Chinese databases. To our knowledge, this is the first systematic review and meta-analysis of meningococcal carriage by both serogroup and age group. An additional strength includes seeking data from authors for meta-analyses. Although not all regions or age groups were able to be analysed, those that were analysed provided initial estimates of the interplay between age and serogroup carriage.

Limitations of this review include the inability to conduct meta-analyses in all regions where data were available, such as in China, and variability in the methodology of the studies included. As discussed above, the study methodology can affect the sensitivity to detect carriage, which, in turn, can affect the results of this review by decreasing the estimated prevalence. Important challenges to this review include the variability of meningococcal epidemiology temporally and geographically. In order to mitigate this limitation, we included studies only from 2007 to 2016 and examined by WHO region. However, we recognise that the epidemiological situation is still variable in this time frame, even year-to-year within the same country. Additionally, neither direct nor indirect vaccine effects were accounted for in the analysis, both of which can have substantial impact on the carriage of the targeted capsular groups. ${ }^{7}$ Limitations notwithstanding, we believe this review will be beneficial to infectious disease specialists, researchers and policy makers who wish to gain a better understanding of the serogroup-specific studies recently conducted and the prominent serogroups carried.

\section{CONCLUSIONS}

Our review provides a thorough evaluation of meningococcal carriage studies from 2007 to 2016 reporting age 
and serogroup data. Due to paucity of data eligible for inclusion in meta-analyses, clear and robust conclusions about the variation of capsular group-specific carriage across all age groups and WHO regions were unable to be determined. The data reported in this review should be used in conjunction with local disease epidemiology when evaluating the ages most responsible for transmission and establishing targeted vaccination policies, as high carriage prevalence does not necessarily correlate with high disease risk. Carriage studies will continue to be important as MenACWY and MenB vaccines are more widely introduced, since their impact on carriage, and the subsequent development of herd immunity, is not yet fully understood. However, this cannot be done effectively if carriage methodology and sensitivity differ substantially between studies. It is, therefore, of great importance that research studies continue to elucidate a standard methodology for carriage detection.

Acknowledgements We would like to thank the MenAfriCar Consortium, Dr LudovicLemée and Dr Lucy McNamara for kindly sharing their data for this review and analyses. We would also like to thank Michael Fane for his assistance in translating the article identified in Persian. Partial results from this review were presented at the European Congress of Epidemiology, Lyon, France, 4-6 July 2018.

Collaborators Meningococcal Carriage Group Members (listed in alphabetical order): Daniela Amicizia (Department of Health Sciences, University of Genoa, Genoa, Italy), Christopher D Bayliss (Department of Genetics and Genome Biology, University of Leicester, Leicester, UK), Leila Carvalho Campos(Laboratório de Patologiae Biologia Molecular, Instituto Gonçalo Moniz, FIOCRUZBAHIA, Salvador, BA, Brazil), Paul Robert Cleary (Public Health England, Field Epidemiology Service, Liverpool, UK), Valérie Delbos (Tropical and Infectious Diseases Department, Rouen University Hospital, Rouen, France), Susanna Esposito (Pediatric Clinic, Department of Surgical and Biomedical Sciences, Università degli Studi di Perugia, Perugia, Italy), Paul A Kristiansen(Department of Bacteriology, Norwegian Institute of Public Health, Oslo,Norway), Sarah A Mbaeyi (National Center for Immunization and Respiratory Diseases, Centers for Disease Control and Prevention, Atlanta, Georgia, USA), Neil J Oldfield (School of Life Sciences, University of Nottingham, Nottingham, UK), Donatella Panatto (Department of Health Sciences, University of Genoa, Genoa, Italy), Fernanda Rodrigues (Faculdade de Medicina, Universidade deCoimbra, Coimbra, Portugal; Hospital Pediátrico, Centro Hospitalar e Universitário de Coimbra, Coimbra, Portugal), Heidi M Soeters (National Centerfor Immunization and Respiratory Diseases, Centers for Disease Control and Prevention, Atlanta, Georgia, USA), David PJ Turner (School of Life Sciences, University of Nottingham, Nottingham, UK; Nottingham University Hospitals NHSTrust, Nottingham, UK) and Aiqiang Xu (Shandong Center for Disease Control and Prevention, Jinan, China).

Contributors HN and MHK conceptualized the study and provided critical comments to the manuscript. MEP led the systematic review; acquired, analyzed, interpreted the data and drafted the manuscript. YL assisted with the systematic review and data interpretation, and provided critical comments to the manuscript. RM and HS assisted with the systematic review and provided critical comments to the manuscript. All members of the Meningococcal Carriage Group contributed data for analysis, assisted with data interpretation and provided critical comments to the manuscript. All authors read and approved the final draft.

Funding This work was supported by Sanofi Pasteur, Inc. MHK is an employee of Sanofi Pasteur, Inc .and had a role in the study conception, data interpretation and preparation of the manuscript. YL is supported by a scholarship from the China Scholarship Council. The China Scholarship Council had no role in this study.

Disclaimer The findings and conclusions in this report are those of the authors and do not necessarily represent the official position of the Centers for Disease Control and Prevention.

Competing interests MHK is an employee of Sanofi Pasteur. CDB reports grants from Sanofi Pasteur for a study that contributed to the analysis, non-financial support from GSK outside of the submitted work, and grants from Roche outside of the submitted work. SE reports grants and personal fees from GSK, Sanofi Aventis, and Merck; grants from Pfizer, Novartis, DMG, and Valeas; and personal fees from
Seqirus outside of the submitted work. FR reports grants paid to a charitable association from Pfizer, GSK, and MSD outside of the submitted work. All other authors have no competing interests to declare. The corresponding author had full access to all the data in the study and had final responsibility for the decision to submit for publication.

Patient consent for publication Not required.

Provenance and peer review Not commissioned; externally peer reviewed.

Data sharing statement No additional unpublished data from this study are available.

Open access This is an open access article distributed in accordance with the Creative Commons Attribution Non Commercial (CC BY-NC 4.0) license, which permits others to distribute, remix, adapt, build upon this work non-commercially, and license their derivative works on different terms, provided the original work is properly cited, appropriate credit is given, any changes made indicated, and the use is non-commercial. See: http://creativecommons.org/licenses/by-nc/4.0/.

\section{REFERENCES}

1. Edmond K, Clark A, Korczak VS, et al. Global and regional risk of disabling sequelae from bacterial meningitis: a systematic review and meta-analysis. Lancet Infect Dis 2010;10:317-28.

2. Pace D, Pollard AJ. Meningococcal disease: clinical presentation and sequelae. Vaccine 2012;30(Supplement 2):B3-9.

3. Harrison LH, Trotter CL, Ramsay ME. Global epidemiology of meningococcal disease. Vaccine 2009;27(Supplement 2):B51-63.

4. Cartwright KA, Stuart JM, Jones DM, et al. The Stonehouse survey: nasopharyngeal carriage of meningococci and Neisseria lactamica. Epidemiol Infect 1987;99:591-601.

5. Cooper L, Kristiansen P, Christensen H, et al. Meningococcal carriage by age in the African meningitis belt: a systematic review and meta-analysis. The European Meningococcal Disease Society. Prague, Czech Republic 2017:120.

6. Christensen H, May M, Bowen L, et al. Meningococcal carriage by age: a systematic review and meta-analysis. Lancet Infect Dis 2010;10:853-61.

7. Borrow R, Alarcón P, Carlos J, et al. The Global Meningococcal Initiative: global epidemiology, the impact of vaccines on meningococcal disease and the importance of herd protection. Expert Rev Vaccines 2017;16:1-16.

8. Trotter CL, Maiden MC. Meningococcal vaccines and herd immunity: lessons learned from serogroup $\mathrm{C}$ conjugate vaccination programs. Expert Rev Vaccines 2009;8:851-61.

9. Vetter V, Baxter R, Denizer G, et al. Routinely vaccinating adolescents against meningococcus: targeting transmission \& disease. Expert Rev Vaccines 2016;15:641-58.

10. The Joanna Brings Institute. The Joanna Briggs Institute critical appraisal tools for use in JBI systematic reviews: checklist for prevalence studies. 2017.

11. Nyaga VN, Arbyn M, Aerts M. Metaprop: a Stata command to perform meta-analysis of binomial data. Arch Public Health 2014;72:39.

12. Peterson ME, Li Y, Bita A, et al. Meningococcal serogroups and surveillance: a systematic review and survey. J Glob Health 2019;9:010409.

13. Trotter CL, Greenwood BM. Meningococcal carriage in the African meningitis belt. Lancet Infect Dis 2007;7:797-803.

14. LaForce FM, Djingarey M, Viviani S, et al. Successful African introduction of a new Group A meningococcal conjugate vaccine: Future challenges and next steps. Hum Vaccin Immunother 2017:1-5.

15. Karachaliou A, Conlan AJ, Preziosi MP, et al. Modeling long-term vaccination strategies with MenAfriVac in the African meningitis belt. Clin Infect Dis 2015;61(Supplement 5):S594-600.

16. Nnadi C, Oladejo J, Yennan S, et al. Large outbreak of neisseria meningitidis serogroup C - Nigeria, December 2016-June 2017. MMWR Morb Mortal Wkly Rep 2017;66:1352-6.

17. Kretz CB, Retchless AC, Sidikou F, et al. Whole-genome characterization of epidemic neisseria meningitidis serogroup c and resurgence of serogroup W, Niger, 2015. Emerg Infect Dis 2016;22:1762-8.

18. Whittaker R, Dias JG, Ramliden M, et al. The epidemiology of invasive meningococcal disease in EU/EEA countries, 2004-2014. Vaccine 2017;35:2034-41.

19. Li J, Shao Z, Liu G, et al. Meningococcal disease and control in China: findings and updates from the Global Meningococcal Initiative (GMI). J Infect 2018;76:429-37. 
20. Attarpour-Yazdi MM, Ghamarian A, Mousaviehzadeh M, et al. Identification of the serotypes of bacterial meningitis agents; implication for vaccine usage. Iran J Microbiol 2014;6:211-8.

21. Borrow R, Lee JS, Vázquez JA, et al. Meningococcal disease in the Asia-Pacific region: findings and recommendations from the Global Meningococcal Initiative. Vaccine 2016;34:5855-62.

22. Roberts J, Greenwood B, Stuart J. Sampling methods to detect carriage of Neisseria meningitidis; literature review. J Infect 2009;58:103-7.

23. Caugant DA, Tzanakaki G, Kriz P. Lessons from meningococcal carriage studies. FEMS Microbiol Rev 2007;31:52-63.

24. Esposito S, Zampiero A, Terranova L, et al. Comparison of posterior pharyngeal wall and nasopharyngeal swabbing as a means of detecting the carriage of Neisseria meningitidis in adolescents. Eur $J$ Clin Microbiol Infect Dis 2013;32:1129-33.

25. Rizek CF, Luiz AM, Assis GR, et al. Comparison of methods to identify Neisseria meningitidis in asymptomatic carriers. Rev Inst Med Trop Sao Paulo 2016;58:58.

26. Manigart O, Okeakpu J, Odutola A, et al. Alternative molecular methods for improved detection of meningococcal carriage and measurement of bacterial density. J Clin Microbiol 2016;54:2743-8.

27. Jeppesen CA, Snape MD, Robinson H, et al. Meningococcal carriage in adolescents in the United Kingdom to inform timing of an adolescent vaccination strategy. J Infect 2015;71:43-52.

28. Gilca R, de Wals P, Costa J, et al. Longitudinal study of Neisseria meningitidis oropharyngeal carriage in adolescents and young adults in Québec City, Canada. The European Meningococcal Disease Society: 20 years EMGM. Austria: Bad Loipersdorf, 2013:64.

29. van Ravenhorst MB, Bijlsma MW, van Houten MA, et al. Meningococcal carriage in Dutch adolescents and young adults; a cross-sectional and longitudinal cohort study. Clinical Microbiology and Infection 2017;23:573.e1-7.

30. Finn A, Morales-Aza B, Sikora P, et al. Density distribution of pharyngeal cazrriage of Meningococcus in healthy young adults: new approaches to studying the epidemiology of colonization and vaccine indirect effects. Pediatr Infect Dis J 2016;35:1080-5.
31. Soeters HM, Whaley M, Alexander-Scott N, et al. Meningococcal carriage evaluation in response to a Serogroup B Meningococcal disease outbreak and mass vaccination Campaign at a CollegeRhode Island, 2015-2016. Clin Infect Dis 2017;64:1115-22.

32. McNamara LA, Thomas JD, MacNeil J, et al. Meningococcal carriage following a vaccination Campaign With MenB-4C and MenB-FHbp in response to a University Serogroup B Meningococcal Disease Outbreak-Oregon, 2015-2016. J Infect Dis 2017;216:1130-40.

33. Breakwell L, Whaley M, Khan UI, et al. Meningococcal carriage among a university student population - United States, 2015. Vaccine 2018;36:29-35.

34. Moreno J, Hidalgo M, Duarte C, et al. Correction: characterization of carriage isolates of neisseria meningitidis in the adolescents and young adults population of Bogota (Colombia). PLoS One 2015;10:e0139983.

35. Delbos V, Lemée L, Bénichou J, et al. Meningococcal carriage during a clonal meningococcal B outbreak in France. Eur J Clin Microbiol Infect Dis 2013;32:1451-9.

36. Rodrigues F, Morales-Aza B, Christensen H, et al. Oropharyngeal carriage of Meningococcus in portugal by group and clonal complex 6 years after adolescent vaccine campaign. Pediatr Infect Dis $J$ 2015;34:1267-9.

37. Gasparini R, Comanducci M, Amicizia D, et al. Molecular and serological diversity of Neisseria meningitidis carrier strains isolated from Italian students aged 14 to 22 years. J Clin Microbiol 2014;52:1901-10.

38. Dolan Thomas J, Hatcher CP, Satterfield DA, et al. sodC-based real-time PCR for detection of Neisseria meningitidis. PLoS One 2011;6:e19361.

39. Jordens JZ, Heckels JE. A novel porA-based real-time PCR for detection of meningococcal carriage. J Med Microbiol 2005;54:463-6.

40. Ali O, Aseffa A, Bedru A, et al. The diversity of meningococcal carriage across the African Meningitis belt and the impact of vaccination with a group A Meningococcal Conjugate Vaccine. $J$ Infect Dis 2015;212:1298-307. 